\title{
COMPARAISON DES ÉQUATIONS DE RÉGRESSION. APPLICATION AU CUBAGE DES PEUPLEMENTS D'ÉPICÉA COMMUN
}

\author{
N. DECOURT \\ Station de Sylviculture et de Production, \\ Centre national de Recherches forestières, I.N.R.A., \\ 54 - Nancy \\ RÉSUMÉ
}

On expose la méthode proposée par KozaK pour comparer les équations de régression (simple ou multiple).

Le but de ce test statistique est de préciser si des équations séparées doivent être utilisées pour chaque population étudiée, ou si toutes les populations peuvent être décrites par une seule équation. On a appliqué cette méthode au calcul des coefficients de forme de peuplements d'Epicéas communs dans cinq régions françaises différentes. Une seule équation est nécessaire. La comparaison des courbes française et allemande (courbe de KrenN) confirme la stabilité de la forme de cette essence.

A la récente réunion du groupe consultatif des statisticiens forestiers (Jouy-enJosas, septembre 1970), A. KozaK a présenté une méthode simple de comparaison des équations de régression.

Cette méthode, d'un intérêt très général, convient parfaitement à la comparaison des tarifs de cubage. Nous l'avons appliquée à l'étude du cubage sur pied des peuplements équiennes d'Epicéa commun (Picea abies (L.) Karst) par la méthode des coefficients de forme.

\section{I. - MÉTHODE DE COMPARAISON DES ÉQUATIONS DE RÉGRESSION}

On dispose de $p$ séries d'observations d'une variable indépendante $y$ et de $m$ variables dépendantes : $x_{1}, x_{2} \ldots x_{j} \ldots x_{m}$. Les $p$ séries comportent chacune $n_{1}, n_{2} \ldots n_{i} \ldots n_{p}$ éléments d'observations. On peut ajuster par la méthode des moindres carrés $p$ équations de régression de la forme :

$$
y=b_{0}+b_{1} x_{1}+\ldots+b_{j} x_{j}+\ldots+b_{m} x_{m}
$$


La seule hypothèse faite par A. KozAK est que les écarts entre les $y$ comparés et les $y$ aiustés constituent pour les $p$ séries d'observation, des populations normales identiques, de moyenne nulle et de même variance $s^{2}$. A. KozaK propose de tester d'abord l'identité des coefficients de régression (hypothèse de parallélisme des $p$ surfaces représentatives des $p$ équations de régression) puis, s'il y a parallélisme, de tester l'identité des $p$ coefficients bo (hypothèse de coïncidence des $p$ surfaces représentatives des équations).

Sa démarche est la suivante :

soit $\mathbf{M}_{i}$ la matrice des produits centrés pour la $\mathrm{i}^{\mathrm{i} \text { me }}$ série d’observations, $\mathbf{B}_{i}$ le vecteur des $m$ coefficients de régression et $\mathbf{D}_{i}$ le vecteur formé par les $m$ sommes des produits centrés de la variable indépendante et des $m$ variables dépendantes. Les coefficients $\mathbf{B}_{i}$ sont solutions du système d'équations bien connu :

$$
\mathbf{M}_{i} \cdot \mathbf{B}_{i}=\mathbf{D}_{i} \text { (équations normales) }
$$

De plus, si $\Sigma_{i} \mathrm{Y}^{2}$ représente la somme des carrés des écarts de la variable dépendante $y$ à sa moyenne $\bar{y}$, la somme des carrés résiduels sera :

$$
(\mathrm{SC})=\Sigma_{i} \mathrm{Y}^{2}-\mathbf{B}_{i} \mathbf{D}_{i}
$$

avec $\left(n_{i}-m-1\right) d^{\circ}$ de liberté.

Pour les $p$ équations, la somme totale des carrés résiduels sera :

$$
\mathrm{SC}=\sum_{i \equiv 1}^{i}(\mathrm{SC})_{i} \text { avec } \sum_{i=1}^{i} n_{i}-m_{p}-p \text { degrés de liberté. }
$$

Si on s'impose la condition suivante :

$\mathbf{B}_{1}=\mathbf{B}_{2}=\ldots \ldots=\mathbf{B}_{i}=\ldots=\mathbf{B}$ (hypothèse de parallélisme), on aura en sommant membre à membre les $\mathrm{P}$ équations normales telles que (1)

$$
\left(\sum_{i=1}^{i=p} \mathbf{M}_{i}\right) \cdot \mathbf{B}=\sum_{i \equiv 1}^{i=1} \mathbf{O}_{i}
$$

B apparaît donc comme la solution de (2) avec une somme des carrés résiduels :

$$
\begin{gathered}
\mathrm{SC}^{\prime}=\sum_{i \equiv 1}^{i}\left(\Sigma_{i} \mathrm{Y}^{2}\right)-\mathbf{B} \cdot \sum_{i \equiv 1}^{i \bar{\Sigma}^{p}} \mathbf{D}_{i} \\
\text { et }\left(\sum_{i \equiv 1}^{i \bar{\Sigma}^{p}} n_{i}-m-p\right) \text { degrés de liberté. }
\end{gathered}
$$

Enfin, si on suppose que les $p$ séries d'observations font partie d'une même population ef qu'on peut ajuster une seule équation à partir des $\mathrm{N}=\sum_{i=1}^{i=p} n_{i} \quad$ éléments d'observations (hypothèse de coïncidence), on ie fera en résolvant le système :

$$
\mathbf{M} . \mathbf{B}=\mathbf{D}
$$

ou M, B, D sont définis comme pour l'équation (1), mais calculés à partir de la totalité des données.

La somme des carrés résiduels sera dans ce cas :

$$
\mathrm{SC}^{\prime \prime}=\mathrm{2} \mathrm{Y}^{2}-\mathbf{B} \quad \text {. D }
$$

( $\Sigma \mathrm{Y}^{2}$ étant la somme des carrés des écarts de $y$ calculés à partir de la totalité des observations),

$$
\operatorname{avec}\left(\sum_{i=1}^{i} n_{i}-p-1\right) \text { degrés de liberté. }
$$


Selon A. KozAK, on peut tester l'hypothèse du parallélisme, en comparant par une analyse de variance les quantités $\mathrm{SC}^{\prime}$ et $\left(\mathrm{SC}^{\prime}-\mathrm{SC}\right)$ (voir tableau 1).

\section{TABLEAU 1. - TABLE 1}

Test du parallélisme

Test for parallelism

\begin{tabular}{|c|c|c|c|c|}
\hline Origine de la variation & d.1. & $\begin{array}{l}\text { Somme } \\
\text { des carrés }\end{array}$ & Carrés moyens & $\mathrm{F}$ \\
\hline $\begin{array}{l}\text { Erreur résiduelle quand on ajuste } \\
p \text { équations séparées } \ldots \ldots \ldots \ldots \\
\text { Erreur résiduelle quand on ajuste } \\
p \text { équations «parallèles } » \ldots \ldots\end{array}$ & $\begin{array}{l}\mathrm{N}-m p-p \\
\mathrm{~N}-m-p\end{array}$ & $\mathrm{SC}$ & $\mathrm{SC}^{\prime}=\mathrm{CM}^{\prime}$ & \\
\hline Différence $\quad \ldots \ldots \ldots \ldots \ldots \ldots \ldots$ & $m(p-1)$ & $\mathrm{SC}^{\prime}-\mathrm{SC}$ & $\begin{array}{l}\mathrm{N}-m-p \\
\frac{\mathrm{SC}^{\prime}-\mathrm{SC}}{m(p-1)}=\mathrm{CM} \mathrm{D}\end{array}$ & $\frac{C M D}{C M^{\prime}}$ \\
\hline
\end{tabular}

Si le parallélisme est vérifié, on peut alors tester l'hypothèse de coïncidence en comparant de la même façon les quantités $\mathrm{SC}^{\prime}$ et $\mathrm{SC}^{\prime \prime}$ - $\mathrm{SC}^{\prime}$ (voir tableau 2).

TABLEAU 2. - TABLE 2

Test de la cö̈ncidence

Test for coincidence

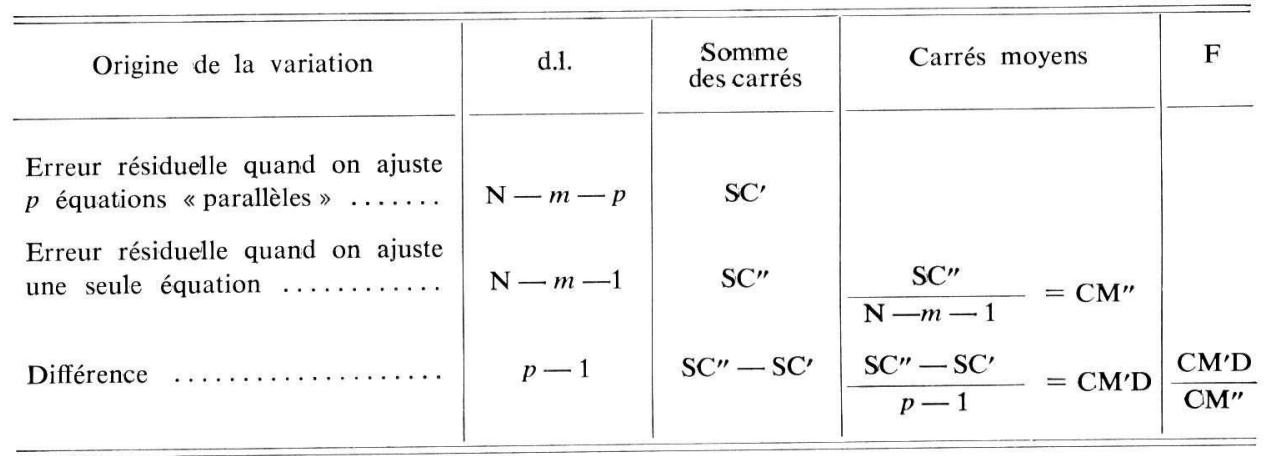

Notons, du reste, qu'on peut également comparer globalement et directement les $p$ équations séparées et une seule équation commune en étudiant les quantités SC et SC" - SC. Cependant, la méthode proposée par A. KozAK apporte plus d'information sur la nature des différences constatées.

\section{II. - CUBAGE SUR PIED DES PEUPLEMENTS FORESTIERS ÉQUIENNES}

J. PARdÉ a montré dans un article de la Revue Forestière Française comment cuber un peuplement sur pied équienne et pur par la formule :

$$
\mathrm{V}=\mathrm{F} \cdot \mathrm{H} \cdot \mathrm{G}
$$


$G$ est la surface terrière du peuplement.

$\mathrm{H}$ est la hauteur moyenne du peuplement (hauteur de la tige de surface terrière moyenne ou mieux hauteur de Lorey, c'est-à-dire moyenne des hauteurs pondérées en surface terrière).

F est le coefficient de forme du peuplement.

$\mathrm{V}$ est le volume du peuplement. Il s'agira ici et par la suite du volume bois-fort (1).

La mesure de $\mathrm{G}$ et de $\mathrm{H}$ permet d'évaluer $\mathrm{V}$ dès lors qu'on connaît la valeur de F. Ce coefficient peut différer sensiblement selon les peuplements, avec l'espèce bien sûr, mais également avec l'âge et la fertilité. Il semble bien établi par exemple que dans un même peuplement, $F$ est petit dans le jeune âge, puis passe par un maximum pour décroître lentement lorsque l'âge augmente. On a rendu compte de ces variations en calculant des relations exprimant $\mathrm{F}$ en fonction de certains paramètres dendrométriques du peuplement et notamment de la hauteur moyenne.

Une bonne méthode pour le faire consiste à étudier la liaison entre le produit $\mathrm{F} \times \mathrm{H}$ (Formhöhe) et la hauteur H. E. Assmann signale que cette relation est très étroite. Il ajoute qu'elle reste stable même pour des traitements sylvicoles très différents.

K. KRENN a précisé cette relation en ajustant des équations de la forme :

$$
\mathrm{FH}=a+b \mathrm{H}+c \mathrm{H}^{2}(a, b \text { et } c \text { sont des constantes })
$$

Pour l'épicéa commun des plaines et collines d'Allemagne occidentale, cette équation prend la forme suivante :

$$
\mathrm{FH}=-2,90+0,87 \cdot \mathrm{H}-0,01 \cdot \mathrm{H}^{2}
$$

On remarquera qu'une telle équation rend bien compte des variations de $\mathrm{F}$, signalées ci-dessus. Lorsque $\mathrm{H}$ crôtt (notamment avec l'âge), F passe par un maximum puis décroît en tendant asymptotiquement vers la droite : $F=0,87-0,01 \mathrm{H}$.

TABLEAU 3. TABLE 3

Résultats des calculs de régression

\begin{tabular}{|c|c|c|c|c|}
\hline Région & $\begin{array}{c}\text { Nombre de } \\
\text { placettes }\end{array}$ & $\begin{array}{l}\text { Carré du } \\
\text { coefficient de } \\
\text { corrélation }\end{array}$ & $\begin{array}{c}\text { Ecart-type } \\
\text { résiduel }\end{array}$ & $\begin{array}{l}\text { Ecart-type } \\
\text { en } \% \text { de la } \\
\text { moyenne }\end{array}$ \\
\hline Basses-Vosges $\ldots \ldots \ldots \ldots \ldots \ldots$ & 99 & 0,946 & 0,503 & 4,7 \\
\hline Plateaux calcaires du N.-E. .... & 28 & 0,918 & 0,648 & 6,1 \\
\hline Haute-Ardèche $\ldots \ldots \ldots \ldots \ldots$ & 33 & 0,949 & 0,566 & 5,4 \\
\hline Limousin $\ldots \ldots \ldots \ldots \ldots$ & 56 & 0,928 & 0,462 & 5,1 \\
\hline Beaujolais-Morvan $\ldots \ldots \ldots \ldots$ & 18 & 0,970 & 0,407 & 3,9 \\
\hline Global .. & 234 & 0,943 & 0,525 & 5,1 \\
\hline
\end{tabular}

Results of regression computations

(1) Volume sur écorce à la découpe de $22 \mathrm{~cm}$ de circonférence. 


\section{III. - APPLICATION AUX PEUPLEMENTS D'ÉPICÉA COMMUN EN FRANCE}

Nous nous sommes servis des résultats obtenus dans les nombreuses placettes semipermanentes installées en France pour diverses études de production et notamment pour la construction de tables de production. Ces placettes ont été regroupées en 5 régiois différentes. On a calculé les équations de KRENN pour chacune de ces régions, ainsi qu'une seule équation à partir de l'ensemble des données.

La méthode de Kozak a été utilisée pour comparer ces 5 équations de régression (tableau 4).

\section{TABlEAU 4. - TABLE 4 \\ Comparaison des régressions \\ Comparison of the regressions}

a) Test du parallélisme.

Test for parallelism.

Origine de la variation

\begin{tabular}{|c|c|c|c|}
\hline d.1. & $\begin{array}{c}\text { Somme } \\
\text { des carrés }\end{array}$ & Carré moyen & $\mathbf{F}$ \\
\hline 227 & 62,10422 & & \\
\hline 219 & $58,17 \cdot 141$ & 0,26562 & \\
\hline 8 & 3,93281 & 0,49160 & $1,85 \mathrm{NS}$ \\
\hline
\end{tabular}

b) Test de la coïncidence.

Test for coincidence.

\begin{tabular}{|c|c|c|c|c|}
\hline Origine de la variation & d.1. & $\begin{array}{c}\text { Somme } \\
\text { des carrés }\end{array}$ & Carré moyen & $\mathbf{F}$ \\
\hline $\begin{array}{l}\text { Erreur résiduelle quand on ajuste } \\
\text { une seule équation } \ldots \ldots \ldots \ldots \ldots\end{array}$ & 231 & 63,63368 & & \\
\hline $\begin{array}{l}\text { Erreur résiduelle quand on ajuste } \\
5 \text { équations «séparées» } \ldots \ldots \ldots\end{array}$ & 227 & 62,10422 & 0,27359 & \\
\hline Différence $\quad \ldots \ldots \ldots \ldots \ldots \ldots$ & 4 & 1,52956 & 0,38237 & $1,40 \mathrm{NS}$ \\
\hline
\end{tabular}

Le test global, qu'on peut facilement déduire du tableau donne $F=1,71$ qui, cela va de soi, n'est pas non plus significatif.

Ajoutons qu'on a vérifié la normalité et l'homogénéité des variances des écarts entre les FH calculés et les FH observés pour les 5 séries d'observations utilisées (tableau 5). 
TABleau 5. - TABle 5

Normalités des écarts

Normality test

\begin{tabular}{l|c|c|c|c|c}
\hline \multicolumn{1}{c|}{ Région } & $\begin{array}{c}\text { Nombre } \\
\text { d'obser- } \\
\text { vations }\end{array}$ & Variance & $\begin{array}{c}\text { Coefficient } \\
\text { de Pearson }\end{array}$ & $\begin{array}{c}\text { Coefficient } \\
\text { de Geary }\end{array}$ & Conclusion \\
\hline Basses-Vosges ..... & 99 & 0,245 & $0,42(1)$ & $0,78 \mathrm{NS}$ & $\begin{array}{l}\text { quasi- } \\
\text { normale. }\end{array}$ \\
$\begin{array}{l}\text { Plateaux calcaires du } \\
\text { N.-E. ......... }\end{array}$ & 28 & 0,364 & $0,23 \mathrm{NS}$ & $0,75 \mathrm{NS}$ & normale. \\
Haute-Ardèche ..... & 33 & 0,290 & $-0,13 \mathrm{NS}$ & $0,81 \mathrm{NS}$ & normale. \\
Limousin ........ & 56 & 0,267 & $0,57(2)$ & $0,79 \mathrm{NS}$ & $\begin{array}{l}\text { quasi- } \\
\text { normale. } \\
\text { Beaujolais-Morvan .. }\end{array}$ \\
\hline
\end{tabular}

(1) 0,39 au seuil 0,05 et 0,57 au seuil 0,01 .

(2) $0,51 \gg 0,05$ et $0,75 \gg 0,01$.

Test de Bartlett: $v=0,263 ; x^{2}=4,82$ N S.

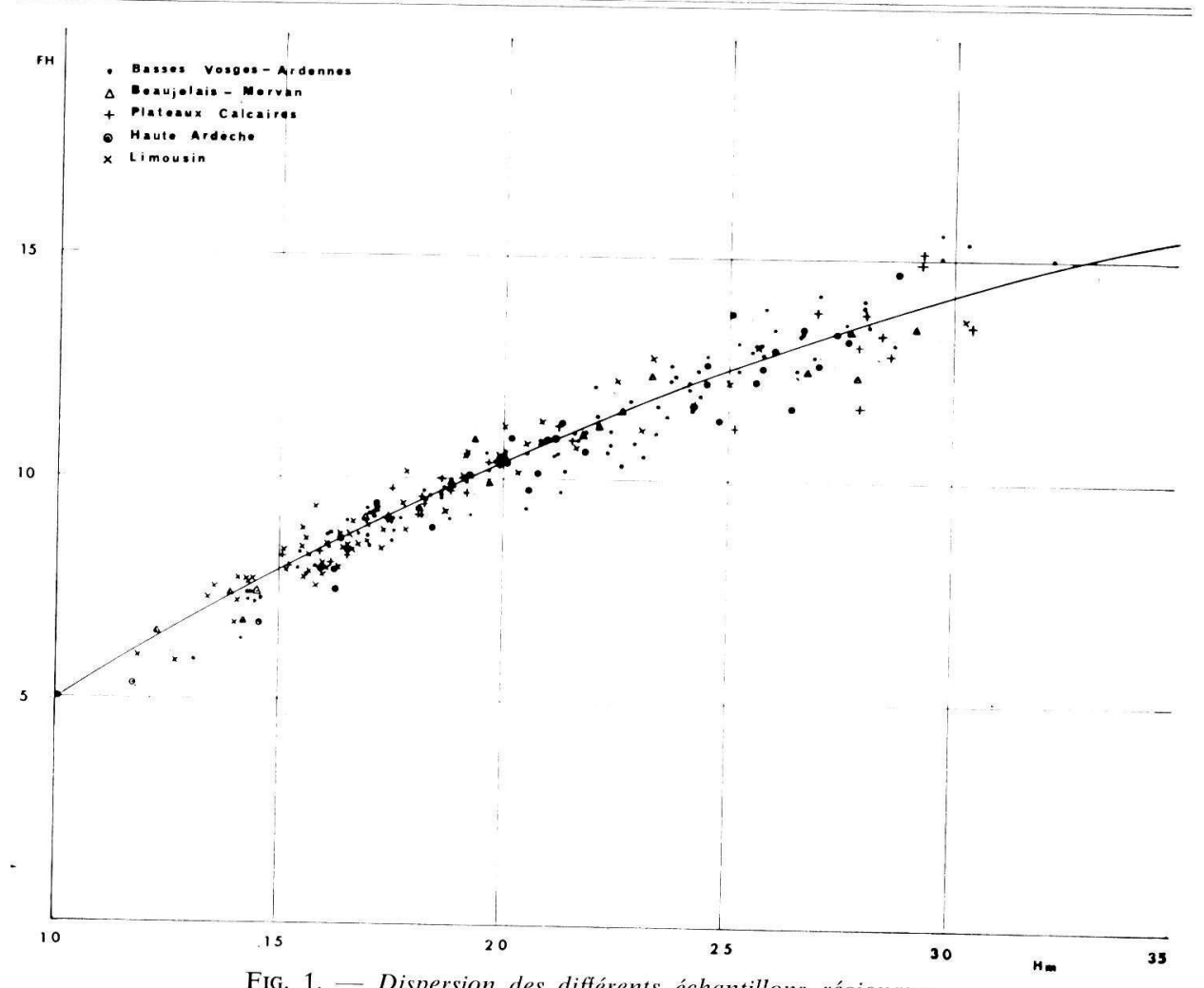

FIG. 1. - Dispersion des différents échantillons régionaux

FIG. 1. - Dispersion of the different regional samples 


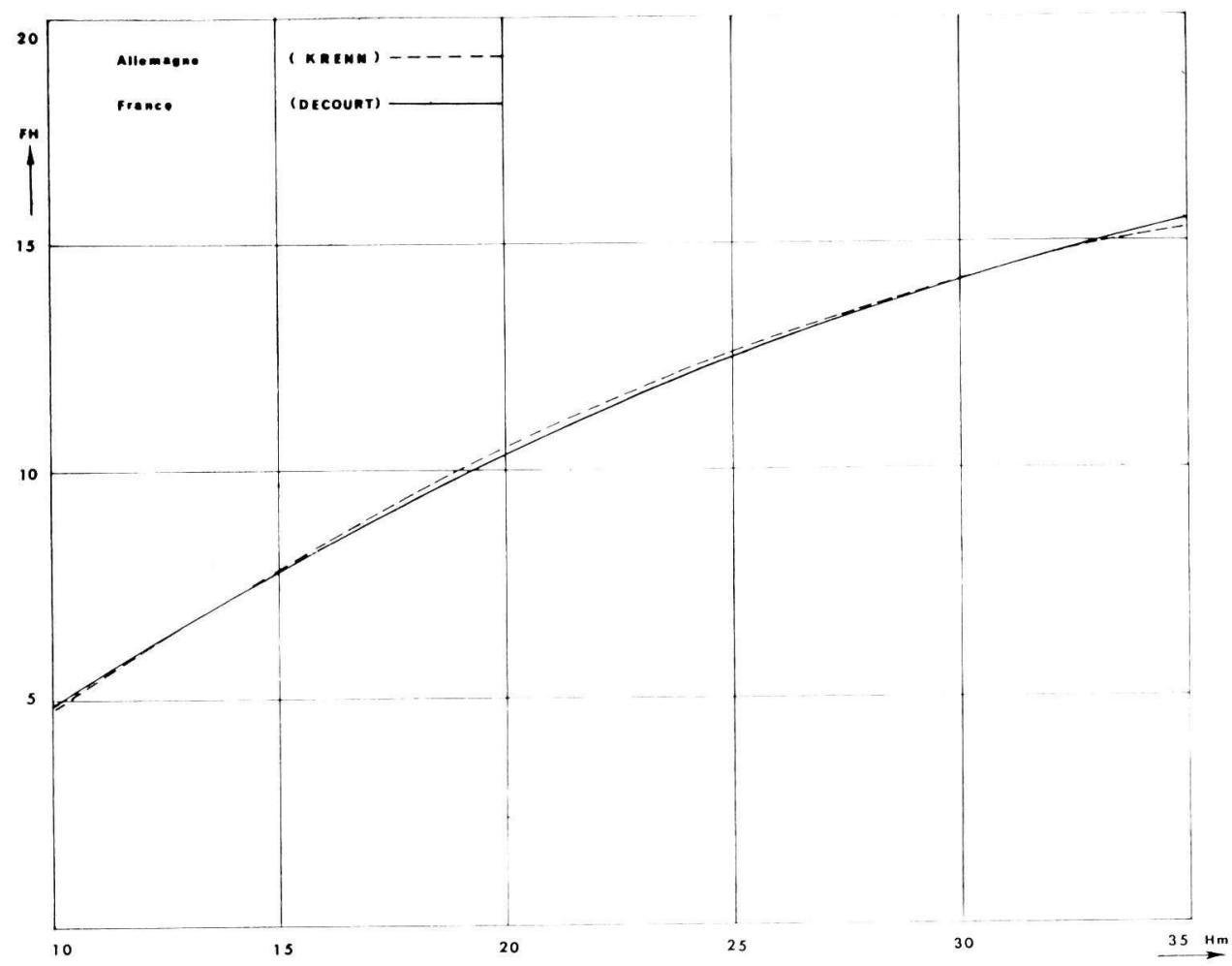

FIG. 2. - Courbe allemande et courbe française

FIG. 2. - German and French curves

L'utilisation de la méthode de KozaK semble licite. On peut donc considérer comme valable dans les cinq régions considérées - et compte tenu de leur dispersion, sans doute dans l'ensemble de la France - l'équation unique ci-dessous obtenue à partir des 234 placettes :

$$
\mathrm{FH}=-2,092+0,37733 \cdot \mathbf{H}-0,00766 \cdot \mathrm{H}^{2}
$$

La figure 1 illustre du reste parfaitement l'existence d'une relation unique commune aux cinq régions étudiées.

On peut essayer également de comparer ce résultat à celui de KrenN. En l'absence de données précises sur les calculs effectués par KRENN, on s'est contenté d'une comparaison graphique (figure 2) qui ne laisse aucun doute sur la très grande similitude des deux relations.

Cette comparaison confirme bien l'extrême stabilité de la «forme » des peuplements d'Epicéa commun dans les p'aines et basses montagnes de l'Europe occidentale. 


\author{
SUMMARY \\ COMPARISON OF REGRESSION EQUATIONS. \\ USE FOR CUBING NORWAY SPRUCE STANDS
}

Kozak's procedure to compare regression equations (simple or multiple) is exposed. The purpose of this statistical test is to determine if separate regression equations should be used for each population under study or if all of the populations could be described by one regression equation. The application to this procedure is made to compute the form coefficient of Norway Spruce stands from five differents parts of France. One equation is sufficient. The comparison of the French curve with the german curve (KrENN) confirme the stability of the form of this species.

\title{
ZUSAMMENFASSUNG
}

VERGLEICHENDE STUDIUM VON VERSCHIEDENEN REGRESSIONSGLEICHUNGEN UND IHRE ANWENDUNG AUF DIE MASSENERMITTLUNG VON FICHTENBESTANDEN

Die von KozaK vergeschlagene Methode zum Vergleich von Einfach-und Mehrfachregressionsgleichungen wird an einem Beispiel dargestellt.

Mit dieser Methode lässt sich feststellen, ob für jede der untersuchten Populationen eine getrennte Regressionsgleichung aufgestellt werden muss, oder alle Populationen durch eine einzige Gleichung beschrieben werden können. Als Beispiel werden die Bestandesformzahlen der Fichte in Fünf verschiedenen Gebieten Frankreichs untersucht. Es zeigts sich dabei, dass nur eine einzige Gleichung benötigt wird.

Ein Vergleich mit der von KRENN für Deutschland aufgestellten Kurve bestätigt die Formstabilität dieser Holzart.

\section{RÉFÉRENCES BIBLIOGRAPHIQUES}

Assmann E., 1961. Waldertragskunde (BLV, München).

KrenN K., 1946. Ertragstafeln f. Fichte. Schriftenr. d. Bad. Forstl. Vers.-Anst., Freiburg.

Pardé J., 1963. Une méthode rapide de cubage approché des peuplements forestiers équiennes. R.F.F. août-sept. 1963 (8-9), 714-720.

KozaK A., 1970 A simple method to tcst parallelism ana coincidence for curvilinear, multiple lincar and multiple curvilinear regressions. 3rd conference of the Advisory Group of Forest Statisticians, section 25, I.U.F.R.O., Jouy-en-Josas, France, sept. 7-11, 1970). 